This document is confidential and is proprietary to the American Chemical Society and its authors. Do not copy or disclose without written permission. If you have received this item in error, notify the sender and delete all copies.

\title{
Through the Lipopolysaccharide Glass: A Potent Antimicrobial Peptide Induces Phase Changes in Membranes
}

\begin{tabular}{|r|l|}
\hline Journal: & Biochemistry \\
\hline Manuscript ID & bi-2016-010632.R1 \\
\hline Manuscript Type: & Article \\
\hline Date Submitted by the Author: & 16-Feb-2017 \\
\hline Complete List of Authors: & $\begin{array}{l}\text { Jefferies, Damien; University of Southampton, Chemistry } \\
\text { Hsu, Pin; University of Southampton, Chemistry } \\
\text { Khalid, Syma; University of Southampton, Chemistry }\end{array}$ \\
\hline
\end{tabular}

SCHOLARONE ${ }^{m}$

Manuscripts 


\title{
Through the Lipopolysaccharide Glass: A Potent Antimicrobial Peptide Induces Phase Changes in Membranes
}

\author{
Author List \\ Damien Jefferies ${ }^{1}$, Pin-Chia Hsu ${ }^{1}$, Syma Khalid ${ }^{1 *}$ \\ ${ }^{1}$ School of Chemistry, University of Southampton, Southampton, SO17 1BJ, UK \\ *to whom correspondence should be addressed
}

\section{Author contributions}

Conceived and designed the experiments: DJ SK. Performed the experiments: DJ. Analysed the data: DJ PCH. Wrote the paper: DJ and SK.

\begin{abstract}
In the following molecular simulations are used to reveal unexpected behavior within bacterial membranes. We show that lipopolysaccharide molecules found in these membranes form viscous amorphous solids when they are interlinked with monovalent and divalent cations. The bilayers exhibit both liquid and glassy characteristics, due to the co-existence of both liquid and crystalline domains in the bilayer. Polymyxin B1 (PMB1), a potent antimicrobial peptide, is shown to increase order within the LPS bilayers by inducing the formation of crystalline patches. Crucially we are able to decompose the energetics of insertion into their enthalpic and entropic components. The present coarse-grain (CG) molecular dynamics (MD) study provides unprecedented insights into the antibacterial action of antimicrobial peptides, thus paving the way for development of novel therapeutic agents to treat multiple drug resistant Gram-negative bacteria.
\end{abstract}




\section{Introduction}

Resistance to antibiotics has increased in recent years, especially among Gramnegative bacteria including Klebsiella pneumonia, Acinetobacter baumannii, and Escherichia coli (E. coli). [1-4] A particular problem is presented by these resilient bacteria proliferating in hospital settings and infecting those with already compromised immune systems. Hampering the search for a solution to this problem is the lack of any new antibiotic agents entering the drug development pipeline. [5-7] The non-availability of new therapies could lead to a post-antibiotic era as the microbes increasingly acquire resistance to our last-line of defence therapies. In part, this dwindling supply of novel antibiotics is ascribed to many large pharmaceutical companies abandoning conventional drug development for more profitable endeavours. [8,9]. While computer simulation, particularly at the molecular level is potentially a powerful tool for the development of targeted and cost-effective novel therapies it is somewhat hampered by our lack of understanding of the molecular pathways utilized by antibiotics to both enter and destroy bacterial cells [10-15]. To further this understanding, simulation of currently used antibiotics with biologically relevant models of bacterial membranes is imperative.

Polymyxin B1 (PMB1) (Fig. 1A), is one of the most potent antimicrobial peptides, that targets Gram-negative bacteria [16-19]. It has been shown to interact with lipopolysaccharide (LPS) molecules, which are the major component of the Gramnegative outer membrane. LPS is a complex macromolecule with a tripartite structure: the highly conserved Lipid A group anchors LPS lipids into the Gram-negative outer membrane, while the anionic core sugars and multifarious glycan polymer portion forms a barrier that protects the bacteria from chemical attack. [20-25] 

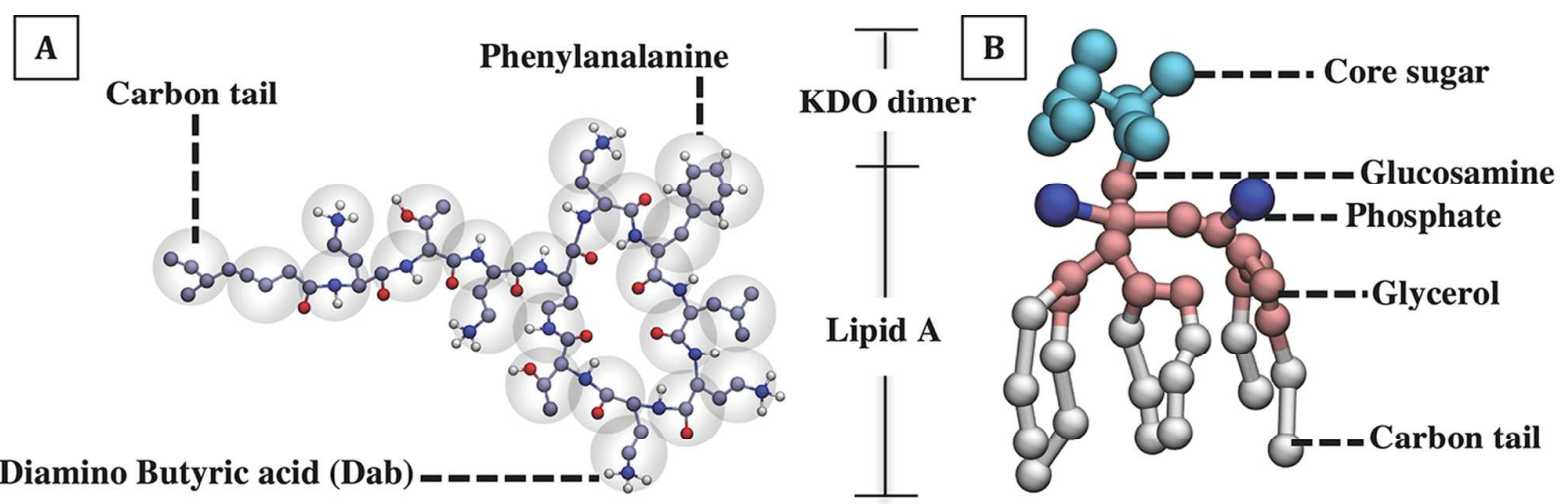

Figure 1. CG models for PMB1 and Re LPS. (A) CG model for PMB1. The Martini beads are shown as translucent spheres, while the underlying united-atom particles are shown as opaque spheres. Carbon atoms are ice blue, oxygen atoms are red, nitrogen atoms are blue, and hydrogen atoms are white. (B) CG model for Re LPS. Carbon tails are white, glucosamine and glycerol groups are pink, phosphate groups are blue, and the remaining core saccharides are cyan. The Re LPS lipid is sectioned into its component Lipid A anchor and 2-keto-3-deoxtoctonoic acid (KDO) dimer for clarity.

In the following, we employ coarse-grain (CG) molecular dynamics (MD) to probe the effect of PMB1 on the local outer membrane environment. We demonstrate that deep-rough (Re) mutants of LPS (Fig. 1B), which contain Lipid A with minimal core oligosaccharide section for bacterial survival (two 2-keto-3-deoxtoctonoic acid units), [26] form bilayers with glass-like properties including high shear viscosities and heterogeneous relaxation dynamics, primarily owing to strong electrostatic interactions between ambient cations and the two anionic phosphate groups of the Re LPS molecules. [27,28] The bilayers are perturbed by PMB1, which affects the dynamics of LPS lipids and thereby induces the formation of small crystalline domains. The crystallization effects are minimized when the Re LPS are linked with divalent cations, as the ions induce close and relatively rigid packing of the lipid head groups, effectively shielding the vulnerable anionic phosphate groups from the polycationic peptides.

\section{Materials}




\section{Molecular dynamics parameters and protocol}

Simulations were performed with the GROMACS simulation package (version 4.5.5) [65] and the Martini force field (version 2.2). [66] The Re LPS bilayers were formed in previous work with the GROMACS genconf utility; each bilayer contained $100 \mathrm{Re}$ LPS molecules per leaflet. [31] The Re LPS membranes (being void of any PMB1 peptides) were equilibrated for $10 \mu \mathrm{s}$ at different temperatures $(300 \mathrm{~K}, 310 \mathrm{~K}$, and 320 K) after initial energy minimization with the steepest decent algorithm. Simulations were run in the NPT ensemble, as the pressure was coupled to 1 bar using a semiisotropic barostat and the Parrinello-Rahman algorithm (time constant $1 \mathrm{ps).} \mathrm{[67,68]}$ Temperature was coupled to a Nosé-Hoover heat bath (time constant of 1 ps). [69] The Lennard-Jones and Coulomb potentials were smoothly reduced to zero between 0 and $1.2 \mathrm{~nm}$ and between 0.9 and $1.2 \mathrm{~nm}$, respectively, using the standard GROMACS shift function. The neighbor list was updated every 10 steps. All simulations were performed with the leapfrog integrator and an integration time step of $10 \mathrm{fs}$. The LPS simulation parameters are validated elsewhere. The PMB1 parameters were optimized with in-house computer code (see supporting information). The concentration of PMB1 peptides was subsequently increased from one, to six molecules per periodic box at the biologically relevant temperature of $310 \mathrm{~K}$. The bilayers that were equilibrated at 300 and $320 \mathrm{~K}$ were used in umbrella sampling simulations alone, to ascertain free energies of interaction between a single PMB1 peptide and a bilayer of Re LPS molecules.

\section{Free energy calculations}

Potential of mean force (PMF) for PMB1 as a function of distance from the bilayer center was determined with umbrella sampling and the weighted histogram analysis method (WHAM) method. [37] The peptide was restrained at a given distance from the bilayer center bilayer using a harmonic potential with force constant of $1000 \mathrm{~kJ}$ $\mathrm{mol}^{-1} \mathrm{~nm}^{-2}$ applied along the bilayer normal; no restraints were applied in the plane of the membrane. The distance between the PMB1 peptide and the bilayer center was varied from $0-4.3 \mathrm{~nm}$, using $0.1 \mathrm{~nm}$ increments. The umbrella sampling simulations were $1 \mu$ s long; simulation parameters were otherwise set to match the unbiased MD simulations. 


\section{Simulation analysis}

Contact analysis was performed with the $g$ _mindist utility, while pairwise radial distribution functions (RDF) were evaluated with the $g \_r d f$ utility. Van Hove correlation functions were determined with the g_vanhove program, while lateral diffusion coefficients were calculated using the $g \_m s d$ utility. Vector field visualization was performed with a recently developed MDAnalysis module. [70] The position of particles along the bilayer normal was tracked with the $g$ traj utility; the configurational entropy was evaluated with the $g \_$covar and $g \_$anaeig programs. The lateral area compressibility moduli were calculated according to equation 4 :

$$
K_{A}=\frac{k_{B} T A_{L}}{N_{L}\left\langle\delta A_{L}^{2}\right\rangle}
$$

where $k_{B}$ is the Boltzmann constant, $T$ is the system temperature, $A_{L}$ is the average surface area per lipid, $N_{L}$ is the number of lipids per membrane leaflet, and $\left\langle\delta A_{L}^{2}>\right.$ is the average of the squared fluctuation of $A_{L}$.

\section{Results}

\section{Effect of ion charge on LPS bilayer properties}

Given that antibiotic permeation processes are affected by the concentration and type of ambient ions, [29,30] we characterized the properties of Re LPS bilayers in the presence of either $\mathrm{Na}^{+}$or $\mathrm{Ca}^{2+}$ ion solutions. Through the use of different static and dynamic bilayer properties we determined that Re LPS bilayers express both fluid and glass-like properties; the carbon tails express fluid characteristics, while their phosphate groups behave like a viscous glass (Fig. S1, S2).

\section{MD simulations of PMB1 peptides with LPS bilayers.}

To evaluate the effect of PMB1 on the properties of Re LPS bilayers, we simulated the peptides with Re LPS bilayers in two different solutions, one containing 
monovalent cations; $\mathrm{Na}^{+}$and one containing divalent cations; $\mathrm{Ca}^{2+}$. The concentrations of the PMB1 peptides are within $50-310 \mu \mathrm{M}$, corresponding to no more than 6 peptides per periodic box $(\sim 13 \times 13 \times 20 \mathrm{~nm})$.

The PMB1 peptides were initially placed $\sim 5 \mathrm{~nm}$ above the Re LPS bilayer center (using their center of mass coordinates for reference). The positively charged Dab residues of PMB1 peptides form stable interactions (defined as a separation distance of $r=0.47 \mathrm{~nm}$ i.e. the effective diameter of a standard CG Martini bead) with the peripheral carboxylate groups of Re LPS after an average time of $60.9 \pm 66.2$ (s.d.) ns, and $80.7 \pm 81.6$ (s.d.) ns for the simulations with $\mathrm{Ca}^{2+}$ and $\mathrm{Na}^{+}$ions, respectively. Thereafter, the PMB1 peptides sporadically traverse the LPS head groups, enabling their Dab residues to interact with the negatively charged Re LPS phosphate groups after an average time of $251.4 \pm 405.8$ (s.d.), and $45.6 \pm 149.9$ (s.d.) ns for the $\mathrm{Ca}^{2+}$ and $\mathrm{Na}^{+}$systems, respectively. The interactions between the PMB1 Dab residues and Re LPS phosphate groups systematically increase during the full $5 \mu$ s of simulation time, but the electrostatic interactions were established far less frequently and rapidly when the Re LPS lipids were linked with divalent $\left(\mathrm{Ca}^{2+}\right)$ ions (Fig 2A, B). Taking the average for all PMB1 molecules throughout the full set of unbiased simulations, the peptides were located at distances of $2.8 \pm 0.6$ and $2.2 \pm 0.6 \mathrm{~nm}$ above the bilayer center during the last $100 \mathrm{~ns}$ of simulation time when the lipids were linked with $\mathrm{Na}^{+}$ and $\mathrm{Ca}^{2+}$ ions, respectively (Fig. 2C, D, S3, S4). The pairwise radial distribution functions (RDFs) have first peak (corresponding to the first coordination shell) values of $\sim 30$ for the interaction between PMB1 Dab residues and peripheral Re LPS carboxylate groups (during this final $100 \mathrm{~ns}$ of simulation time) regardless of ambient ion type, while the first peak RDF values were $\sim 15$ and $\sim 60$ for the interaction between Dab residue side chain and Re LPS phosphate groups in the presence of $\mathrm{Ca}^{2+}$ and $\mathrm{Na}^{+}$ions, respectively (Fig. 2E-H). Thus, the $\mathrm{Ca}^{2+}$ ions induce close and rigid packing of the Re LPS head groups relative to $\mathrm{Na}^{+}$ions, more effectively shielding the vulnerable anionic phosphate groups from the polycationic PMB1 peptides. 
Figure 2. Progressive membrane association of PMB1 depends on ambient ion phosphate groups as a function of simulation time. The cut-off distance was $0.47 \mathrm{~nm}$ (the effective size of a CG Martini bead); data were collated for all unbiased simulations. (A) Data for the bilayers loaded with $\mathrm{Na}^{+}$ions; (B) data for the bilayers loaded with $\mathrm{Ca}^{2+}$ ions. (C, D) Final-frame snapshots show PMB1 peptides (green) interacting with Re LPS bilayers in the presence of (C) $\mathrm{Na}^{+}$or (D) $\mathrm{Ca}^{2+}$ ions. Re LPS lipids are colored according to Fig. $1 \mathrm{~B} ; \mathrm{Na}^{+}, \mathrm{Ca}^{2+}$, and water particles are omitted for clarity. (E-H) RDFs for the phosphate (black lines) and carboxylate (blue lines) groups of Re LPS with respect to the PMB1 Dab residue side chains in the presence of $(\mathrm{E}, \mathrm{F}) \mathrm{Na}^{+}$, or $(\mathrm{G}, \mathrm{H}) \mathrm{Ca}^{2+}$ ions (data were sampled for the last $100 \mathrm{~ns}$ of simulation time, where the simulations were completely converged, or close to equilibrium values). Simulations were performed at $310 \mathrm{~K}$ (to mimic conditions in vivo).

In either instance, there is minimal insertion of the PMB1 peptides into the Re LPS lipid core. Overall, we observed only two isolated incidents of the hydrophobic side chains and lipid tail completely bypassing the Re LPS core sugars and phosphate group to reach the bilayer interior. In the first case, the benzyl (Fig. 3A, S5) and 
isobutyl groups penetrated the phosphate interface of an Re LPS bilayer that was loaded with $\mathrm{Ca}^{2+}$ ions, while in the second case, a single PMB1 carbon tail circumvented the phosphate interface of an Re LPS bilayer that was loaded with $\mathrm{Na}^{+}$ ions. The infrequency of these complete insertion events can be rationalized via twodimensional Voronoi tessellations of the LPS phosphate groups, (Fig. 3B) coupled with calculations of lateral area compressibility moduli. Polygon-based tessellation of the LPS phosphate groups revealed that the PMB1 peptides follow the same translocation mechanism predicted for $\mathrm{C}_{60}$ nanoparticles into lipid bilayers. [31-33] The hydrophobic groups enter the bilayer core as the phosphate groups transiently form small micropores in the membrane surface. As the hydrophobic moieties enter these transient micropores they expand the local defects, enabling their gradual penetration into the bilayer core. (Fig. 3C) The lateral area compressibility moduli $\left(K_{\mathrm{A}}\right)$ for the Re LPS membranes were $1.700 \pm 0.003$ and $0.673 \pm 0.002 \mathrm{~N} \mathrm{~m}^{-1}$ for the bilayers with $\mathrm{Ca}^{2+}$ and $\mathrm{Na}^{+}$ions respectively (calculated at $310 \mathrm{k}$ ), which are larger than the compressibility moduli calculated for conventional DOPC lipid membranes from CG simulations at $300 \mathrm{~K}\left(0.371 \pm 8 \mathrm{~N} \mathrm{~m}^{-1}\right)$ using the Martini force-field, [34] or for DMPC and DPPC lipid bilayers in X-ray scattering studies $\left(0.234\right.$ and $0.231 \mathrm{~N} \mathrm{~m}^{-}$ 1 , respectively). [35,36] The combination of slow Re LPS dynamics, thick hydrophilic core saccharide sections, and high lateral area compressibility moduli contribute to making the process of bilayer penetration rather slow. Indeed, despite our microsecond timescale simulations, it is worth bearing in mind that the final lipidpeptide conformations likely do not represent global energy minima. 


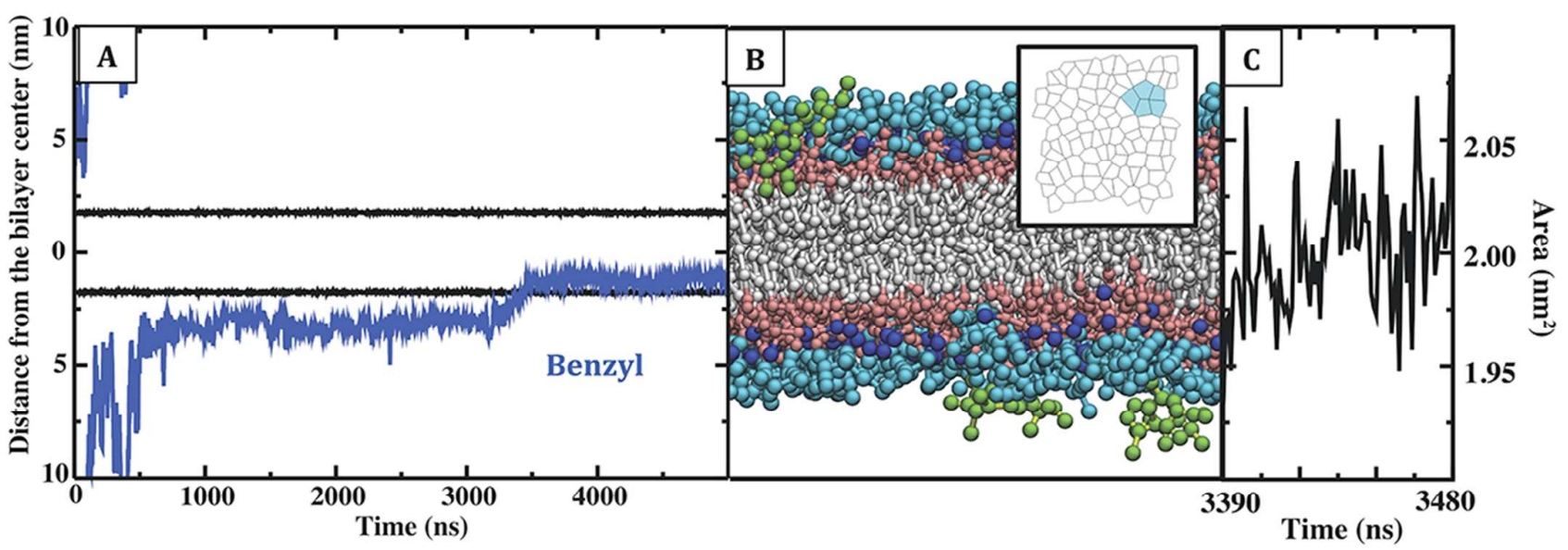

Figure 3. PMB1 benzyl group penetrates the lipid core. (A) Positions of Re LPS phosphate groups and a representative PMB1 benzyl group are shown as black and blue lines, respectively. The coordinates are with respect to the bilayer normal; distances are relative to the bilayer center. The temperature was $310 \mathrm{~K}$; pressure was 1 bar; ambient ions were $\mathrm{Ca}^{2+}$ ions. The center of mass coordinates for all PMB1 peptides in this simulation are shown in Fig. S5. (B) Side-view snapshot for the insertion event; perspective is reversed relative to (A) for clarity. The inset shows the two-dimensional Voronoi tessellation for Re LPS head groups as PMB1 enters the lipid core; projected polygons are colored cyan if they represent lipids adjacent to the embedded PMB1 benzyl group. (C) Area per lipid for the Re LPS lipids adjacent to the benzyl group during part of this insertion event (3390-3480 ns); for comparison, the bilayer average was $1.60 \pm 0.004 \mathrm{~nm}^{2}$.

Free energy, enthalpy and entropy of PMB1 insertion into LPS bilayers.

Given the timescale caveat mentioned above due to the slow process of membrane insertion, we have constructed potential of mean force profiles (PMFs) using umbrella-sampling simulations combined with the weighted histogram analysis method (WHAM) to characterize the energetics of PMB1 penetration into the Re LPS lipid core. [37] A similar study has recently been reported for small molecule permeation through an asymmetric model of the outer membrane. [38] The PMF profiles for PMB1 permeation across Re LPS bilayers at temperatures of 300, 310, and $320 \mathrm{~K}$ are presented in Fig. 4A, B. Notably, the PMF energy minima are located approximately 1.6 and $1.9 \mathrm{~nm}$ from the bilayer center for the systems with $\mathrm{Ca}^{2+}$ and $\mathrm{Na}^{+}$ions, which is 1.2 and $0.2 \mathrm{~nm}$ smaller than the separation distance between PMB1 
peptides and the Re LPS bilayer center calculated using the last $100 \mathrm{~ns}$ of the unbiased MD simulations. At the heights corresponding to the PMF energy minima, PMB1 can interact with the negatively charged carboxylate and phosphate groups of the Re LPS lipids, while the peptide hydrophobic side chains and acyl tail slot into the interstitial gaps in the membrane core.
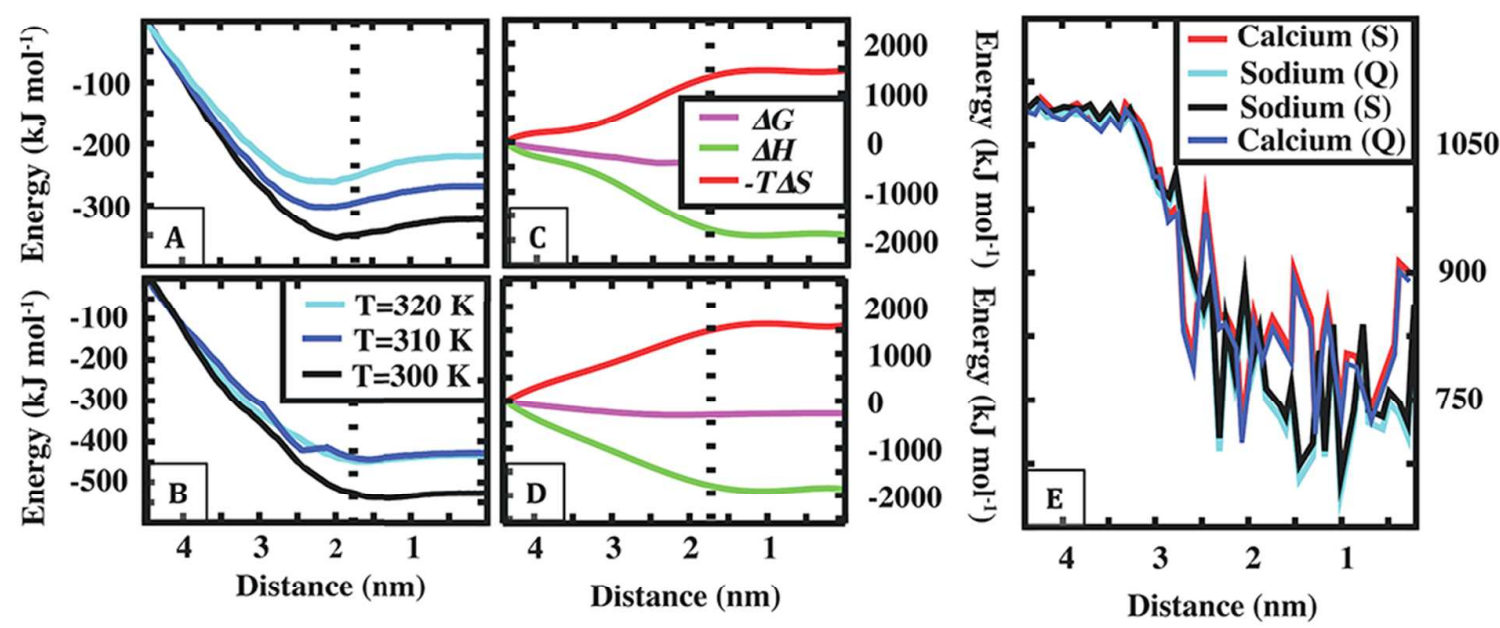

Figure 4. Free energy of PMB1 translocation. Free energies for systems with $(A, C)$ $\mathrm{Na}^{+}$or (B, D) $\mathrm{Ca}^{2+}$ ions. (A, B) PMF profiles for PMB1 as a function of distance from the bilayer center for system temperatures: $320 \mathrm{~K}$ (solid cyan lines), $310 \mathrm{~K}$ (solid blue lines), and $300 \mathrm{~K}$ (solid black lines). (C, D) Free energies computed at $310 \mathrm{~K} \Delta G$ were decomposed into entropic $-T \Delta S$ (solid red lines) and enthalpic $\Delta H$ (solid green lines) components. The dashed black lines show the average position of Re LPS phosphate groups from the bilayer center. (E) The configurational entropy for PMB1 was evaluated with the Schlitter formula (S) and quasi-harmonic approximations (Q) as a function of distance from the bilayer center. Cyan and black lines show data for the simulations with $\mathrm{Na}^{+}$ions; red and blue lines show the data for simulations with $\mathrm{Ca}^{2+}$ ions.

Additional details, including the enthalpic and entropic contributions to the calculated free energy values, can be extracted from the PMF profile temperature dependence:

$$
-T \Delta S=T \frac{d G}{d T} \approx \frac{T}{2 \Delta T}(G(T+\Delta T)-G(T-\Delta T))
$$




$$
\Delta H=\Delta G+T \Delta S
$$

where $G$ is the Gibbs free energy, $H$ is the enthalpy, $T$ is the system temperature and $S$ is entropy. [39] The decomposition of the free energies reveals that PMB1 penetration is driven by a favourable change in enthalpy, but is hindered by an unfavourable change in system entropy (Fig. 4C, D). To evaluate the origins of this entropic wall we have evaluated the configurational entropy of PMB1 as a function of peptide distance from the bilayer center, using the Schlitter formula, [40] and the quasiharmonic approximation (Fig. 4E). [41] The analysis reveals that the configurational entropy of PMB1 decreases by $\sim 0.3 \mathrm{~kJ} \mathrm{~mol}^{-1} \mathrm{~K}^{-1}$ as the peptide moves from bulk solvent to lipid core, demonstrating that the drastic changes in system entropy cannot be ascribed to changes in PMB1 conformation alone; the calculations suggest that the entropy of the membrane must also be changing.

To determine if the PMB1 peptide was affecting the dynamical characteristics of the Re LPS molecules, and thereby the membrane entropy, we evaluated the self-part of the van Hove correlation function for the leaflet of Re LPS phosphate groups in contact with PMB1. The self-part of the van Hove correlation function provides the probability that a given particle moves a distance $r$ from its neighboring particles within a time interval $t$. The metric has been used previously to quantify both rotational and translational lipid motions in CG simulations, where the quantity helped to clarify the differences between the gel phase and fluid phase of DPPC bilayers. [42] In addition, we monitored the movement of individual Re LPS phosphate groups and analysed the phase characteristics of the phosphate plane via two-dimensional Voronoi analysis. There are marked differences in the van Hove correlation functions (relative to the peptide free simulations) as the Re LPS lipids interact with PMB1 (Fig. 5, S6). There is a shift from clustered continuous-time random walk processes, which are typical of glassy systems, to localized oscillatory and rattling motions, comparable to the trajectories of ions in rigid crystals. $[43,44]$ This is also evident from Re LPS phosphate group lateral diffusion coefficients, which decrease by several orders of magnitude as PMB1 moves from bulk solvent into the bilayer core (Fig. S7). The origins of the differing relaxation dynamics can be explored through two-dimensional Voronoi decompositions of the Re LPS phosphate 
groups. The triangulations suggest that PMB1 is inducing the formation of small, ordered domains (Fig. 6A, B) similar to the crystalline patches observed in experimental studies of LPS from rough strains of E. coli. $[45,46]$ The Voronoi polygons have projected surface areas of $\sim 0.5-0.7 \mathrm{~nm}^{2}$ (Fig. 6D) per phosphate, of which there are two per Re LPS molecule, matching area per phosphate group values $\left(0.55 \mathrm{~nm}^{2}\right)$ obtained for nanocrystalline domains in experiment. Additionally, the phosphate groups have an average of three Re LPS neighbors (corresponding to six anionic phosphate groups) when they form these crystalline patches (Fig. 6F), matching the hexagonal coordination of the Re LPS crystalline domains identified in the grazing incidence X-ray diffraction experiments. $[45,46]$ The crystalline domains appear to be stable for several hundreds of nanoseconds (Fig. S8).
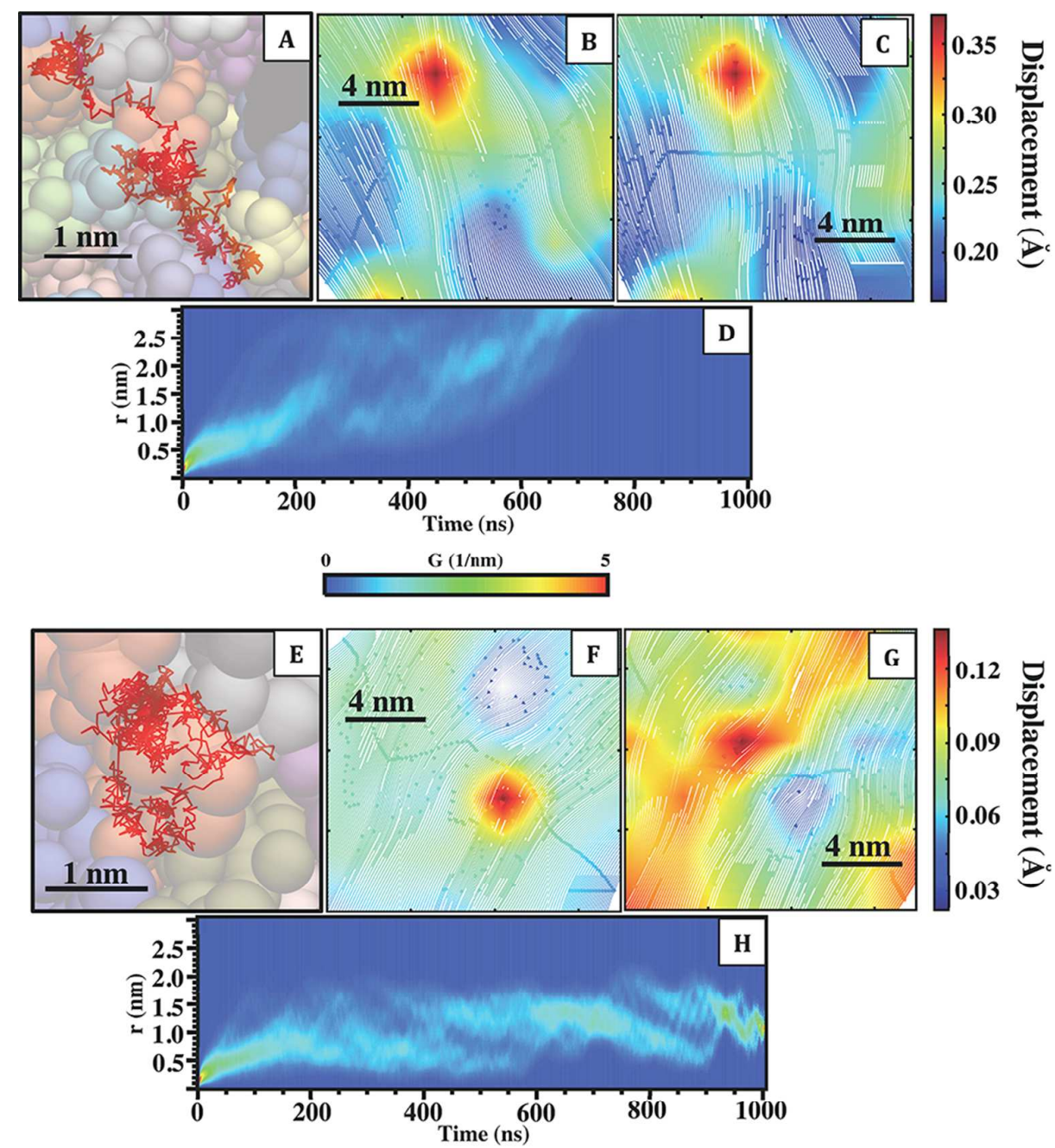

Figure

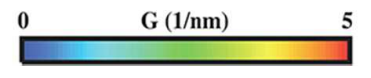

5. Diffusion of Re LPS is affected by PMB1 peptides. (A, E) The trajectories of 
single representative Re LPS phosphate groups were visualized over the course of 1 $\mu \mathrm{s}$ umbrella sampling simulations, the sampled windows correspond to the minimum of the PMF profiles that were calculated at $310 \mathrm{~K}$ (see Fig. 4A, B for reference). The trajectories of the representative phosphate groups is depicted as a red line; final frame Re LPS lipids are shown in the background using different colors to provide a sense of scale and clarity. (A) For the bilayer loaded with $\mathrm{Na}^{+}$ions, there is a shift away from the clustered-continuous-time-random walk processes (see Fig. S6A for comparison), which are typical of glassy systems and a transition towards the localized oscillatory and rattling motions, more similar to the trajectories of ions in rigid crystals $[43,44]$. (E) When the PMB1 peptide is included in the Re LPS bilayer loaded with $\mathrm{Ca}^{2+}$ ions, there is a more significant shift away from clusteredcontinuous-time-random walk processes (see Fig. S6E for comparison) towards localized oscillatory motions, with phosphates confined to domains of $\sim 2 \mathrm{~nm}^{2}$ during $1 \mu$ s of simulation time. (B-C, F-G) Streamline visualization of representative simulation frames to clarify the collective Re LPS head group relaxation dynamics in the presence of a single PMB1 peptide. The collective motion significantly departs from the relaxation dynamics of Re LPS phosphate groups in the absence of any antimicrobial peptides (see Fig. S6B-C, F-G for comparison). There is an approximate order of magnitude reduction in the head group displacements per simulation step (see adjoining color bars for clarity). (D, H) Self-part of the van Hove correlation function, which is the probability that a particle traverses a particular distance within a given timeframe, for Re LPS phosphate groups. Comparisons between Fig. S6D, S6H and Fig. 5D, 5H, respectively reveal significant differences in the relative mobility of Re LPS molecules when they interact with a PMB1 peptide regardless of ion type loading.

Further proof for the inferred crystallization effects comes from calculated heat capacity change profiles, which were determined according to equation 3 , following the protocol in [39]

$$
\Delta C_{p}=-T \frac{d^{2} G}{d T^{2}} \approx \frac{T}{\Delta T^{2}}(\Delta G(T-\Delta T)-2 \Delta G(T)+\Delta G(T+\Delta T))
$$


the heat capacity change becomes more negative as PMB1 traverses the LPS head groups and much of the lipid core (Fig. 6G, H), inline with transitions from disordered to ordered states in amorphous solid materials. $[47,48]$ In this respect, our CG simulations agree with previous united-atom simulation studies in showing that PMB1 has little affect on the average structural properties of LPS containing membranes, [10] but rather exerts important changes for the diffusive properties of LPS lipids that fundamentally alters their trajectories and phase behaviour. The consistent lack of any noticeable mechanical bilayer damage (e.g. bilayer rupture, micellization, formation of pores) or modification of average membrane properties including total projected surface area and membrane thickness in unbiased MD simulations (Table $\mathrm{S} 1$ ), suggests that significant structural changes to lipid bilayers are not responsible for the antimicrobial action of PMB1, but rather that modifications to the diffusive properties of these lipids disrupts Gram-negative membranes.

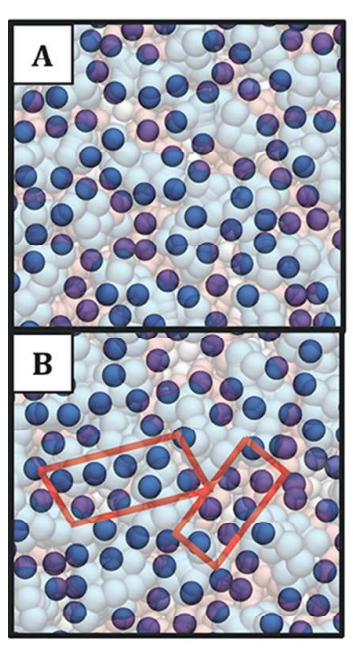

Area per phosphate $\left(\mathrm{nm}^{2}\right)$

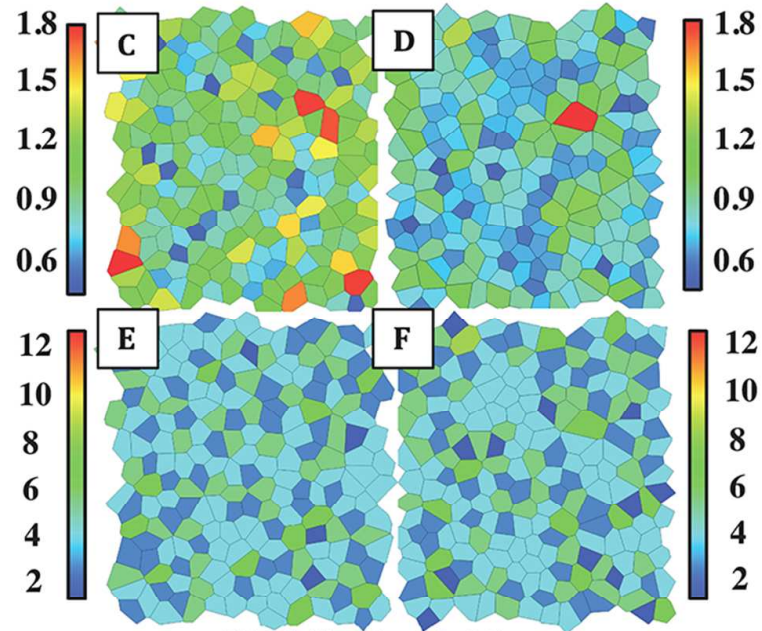

LPS lipid neighbors
Distance (nm)

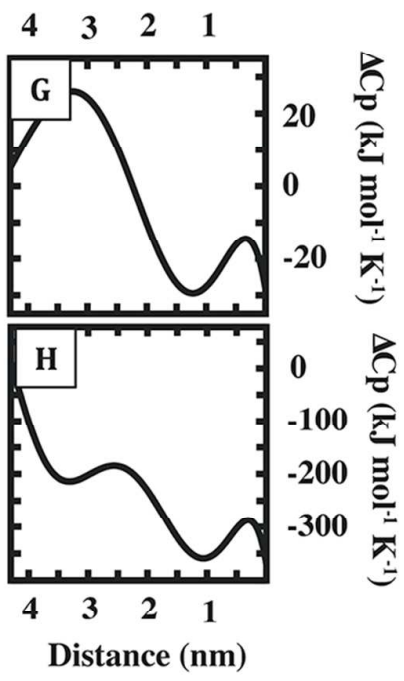

Figure 6. Peptide induced glass-to-crystal transformation. (A, B) Top-view snapshots for Re LPS bilayers in the (A) absence and (B) presence of a PMB1 peptide. Re LPS phosphate groups are shown as opaque blue spheres, while the remaining components of the Re LPS molecules are depicted as translucent spheres. Red quadrilaterals highlight emerging crystalline domains. The bilayers were loaded with $\mathrm{Na}^{+}$ions, the simulation temperature was $310 \mathrm{~K}$, and the (C-F) Voronoi tessellations for Re LPS phosphate groups in the $(\mathrm{C}, \mathrm{E})$ absence and $(\mathrm{D}, \mathrm{F})$ presence of the PMB1 peptide; (D, F) figures were formed by sampling data from the umbrella 
window that corresponds to the minimum of the PMF profile calculated at $310 \mathrm{~K}$ (see Fig. 4A for reference). (C, D) Plots show the area per phosphate, of which there are two per individual Re LPS molecule (see adjoining color scale bars for clarity). (E, F) Plots show the number of whole Re LPS lipids neighboring the tessellated Voronoi cells (see adjoining color scale bars for clarity). (F) The number of 3 Re LPS neigbors (corresponding to 6 phosphate groups), is indicative of hexagonal packing, which has previously been observed in experimental studies, when the bilayers are strained or placed under high surface pressures. [45] $(\mathrm{G}, \mathrm{H})$ Heat capacity change as a function of distance between PMB1 and the bilayer center, data are for the systems with $(\mathrm{G}) \mathrm{Na}^{+}$ and $(\mathrm{H}) \mathrm{Ca}^{2+}$ ions.

\section{Discussion}

Molecular Dynamics simulations at a coarse-grain resolution were used to study the perturbation of LPS bilayers by PMB1 peptides. The simulations revealed that Re LPS lipids move according to complex heterogeneous relaxation dynamics that are a hallmark of glassy systems. $[27,28]$ Similarly, their phosphate groups have properties of amorphous solids including high shear viscosities, radial distribution functions characteristic of the vitreous state, and normalized velocity autocorrelation functions that that are marked by long term oscillations. [49-52] As PMB1 was moved from bulk $\mathrm{Na}^{+}$or $\mathrm{Ca}^{2+}$ ionic solvent to Re LPS bilayer core, it decreased the entropy of the Re LPS lipids, most noticeably in the phosphate plane, and thus perturbed their dynamics. The phosphates were increasingly localized as they were affected by PMB1, making the plane of particles less like an amorphous solid, and more similar to a solid crystal. Indeed, the phosphate groups took on an altogether different arrangement in the presence of PMB1, with small crystalline domains emerging that match microscopic crystallites observed in rough mutant LPS monolayers affected by high surface pressure in experimental studies. $[45,46]$ Notably, these crystallization effects depend on the ability of PMB1 peptides to circumvent the protective Re LPS core sugars, and thereby attack the negatively charged Re LPS phosphate groups.

The full biological implications of such phase effects in the outer membrane are not immediately apparent, but the pertinence of lipid fluidity to important membrane processes and properties is well known. Small changes in lipid order and fluidity can 
drastically alter the physical properties of bilayers including elastic moduli, shape, mechanical stress and tension, [53-56] and can also influence important membrane processes that affect cell viability including protein sorting, signal transduction, molecular transport, enzymatic activities and immune responses. [57-61] Thus, we tentatively propose that the antibacterial action of PMB1 rather than being simply due to direct mechanical bilayer damage and cytoplasmic membrane permeabilization, which has been proposed for many antibiotics that are effective against Gram-positive bacteria, $[62,63]$ or disruptive membrane processes that have been proposed for PMB1, such as the displacement of stabilizing ions, [64] but is also a product of its ability to induce phase changes in the local membrane environment.

\section{Supporting Information}

The static and dynamic properties of Re LPS bilayers were quantified when they were loaded with either monovalent sodium $\left(\mathrm{Na}^{+}\right)$ions, or divalent calcium $\left(\mathrm{Ca}^{2+}\right)$ ions. The analyses shows that the carbon tails express fluid characteristics, while the anionic phosphate groups (of Re LPS) behave like a viscous glass. Additionally information is provided for the CG parameterization of PMB1.

\section{Acknowledgments}

This project made use of time on ARCHER granted via the UK High-End Computing Consortium for Biomolecular Simulation, HECBioSim (http://hecbiosom.ac.uk), supported by EPSRC (grant no. EP/L000253/1). James Graham developed in-house computer code.

\section{References}

1. Hawkey PM, Jones AM. (2009) The Changing Epidemiology of Resistance. J. Antimicrob. Chemother. 64(1): i3-i10.

2. McGowan JE. (2006) Resistance in Nonfermenting Gram-Negative Bacteria: Multidrug Resistance to the Maximum. Am. J. Med. 119(6): S29-S36

3. Neuhauser MM, Weinstein RA, Rydman R, Danziger LH, Karam G, Quinn JP. (2003) Antibiotic Resistance Among Gram-Negative 
Bacilli in US Intensive Care Units: Implications for

Fluoroquinolone Use. JAMA. 289(7): 885-888.

4. Johnson JR, Johnston B, Clabots C, Kuskowski MA, Castanheira

M. (2010) Escherichia coli Sequence Type ST131 as the Major

Cause of Serious Multidrug-Resistant E. coli Infections in the

United States. Clin. Infect. Dis. 51(3): 286-294.

5. Siegel RE. (2008) Emerging Gram-Negative Antibiotic Resistance:

Daunting Challenges, Declining Sensitivities, and Dire

Consequences. Respir. Care 53(4): 471-479.

6. Falagas ME, Bliziotis IA. (2007) Pandrug-Resistant Gram-Negative

Bacteria: the Dawn of the Post-Antibiotic Era? Int. J. Antimicrob.

Ag. 29(6): 630-636.

7. Livermore DM. (2004) The Need for New Antibiotics. Clin.

Microbiol. Infect. 10(4): 1-9.

8. Katz ML, Mueller LV, Polyakov M, Weinstock SF. (2006) Where

Have All the Antibiotic Patents Gone? Nat. Biotechnol. 24(12):

1529-1531.

9. Norrby SR, Nord CE, Finch R. (2005) Lack of Development of

New Antimicrobial Drugs: a Potential Serious Threat to Public

Health. Lancet Infect. Dis. 5(2): 115-119.

10. Berglund NA, Piggot TJ, Jefferies D, Sessions RB, Bond PJ, Khalid

S. (2015) Interaction of the Antimicrobial Peptide Polymyxin B1

with Both Membranes of E. coli: a Molecular Dynamics Study.

PLoS Comput. Biol. 11(4): e1004180.

11. Nawae W, Hannongbua S, Ruengjitchatchawalya M. (2014)

Defining the Membrane Disruption Mechanism of Kalata B1 via

Coarse-Grained Molecular Dynamics Simulations. Sci. Rep. 4:

3933.

12. Polyansky AA, Ramaswarny R, Volynsky PE, Sbalzarini IF, Marrink SJ, Efremov. (2010) Antimicrobial Peptides Induce Growth of Phosphatidylglycerol Domains in a Model Bacterial Membrane. J. Phys. Chem. Lett. 1(20): 3108-3111. 
13. Horn JN, Romo TD, Grossfield A. (2013) Simulating the Mechanism of Antimicrobial Lipopeptides with All-Atom Molecular Dynamics. Biochemistry 52(33): 5604-5610.

14. Bocchinfuso G, Palleschi A, Orioni B, Grande G, Formaggio F, Toniolo C, Park Y, Hahm KS, Stella L. (2009) Different Mechanisms of Action of Antimicrobial Peptides: Insights from Fluorescence Spectroscopy Experiments and Molecular Dynamics Simulations. J. Pep. Sci. 15(9): 550-558.

15. Sengupta D, Leontiadou H, Mark AE, Marrink SJ. (2008) Toroidal Pores Formed by Antimicrobial Peptides Show Significant Disorder. Biochim. Biophys. Acta 1778(10): 2308-2317.

16. Evans ME, Feola DJ, Rapp RP. (1999) Polymyxin B Sulfate and Colistin: Old Antibiotics for Emerging Multiresistant GramNegative Bacteria. Ann. Pharmacother. 33(9): 960-967.

17. Gales AC, Reis AO, Jones RN. (2001) Contemporary Assessment of Antimicrobial Susceptibility Testing Methods for Polymyxin B and Colistin: Review of Available Interpretative Criteria and Quality Control Guidelines. J. Clin. Microbiol. 39(1): 183-190.

18. Sobieszczyk ME, Furuya EY, Hay CM, Pancholi P, Della-Latta P, Hammer SM, Kubin CJ. (2004) Combination Therapy with Polymyxin B for the Treatment of Multidrug-Resistant GramNegative Respiratory Tract Infections. J. Antimicrob. Chemother. 54(2): 566-569.

19. Viljanen P, Vaara M. (1984) Susceptibility of Gram-Negative Bacteria to Polymyxin B Nonapeptide. Antimicrob. Agents Chemother. 25(6): 701-705.

20. Caroff M, Karibian D, Cavaillon JM, Haeffner-Cavaillon N. (2002) Structural and Functional Analyses of Bacterial Lipopolysaccharides. Microb. Infect. 4(9): 915-926.

21. Erridge C, Bennett-Guerrero E, Poxton IR. (2002) Structure and Function of Lipopolysaccharides. Microb. Infect. 4(8): 837-851.

22. Caroff M, Karibian D. (2003) Structure of Bacterial Lipopolysaccharides. Carbohydr. Res. 338(23): 2431-2447. 
23. Zähringer U, Lindner B, Rietschel ET. (1993) Molecular Structure of Lipid A, the Endotoxic Center of Bacterial Lipopolysaccharides. Adv. Carbohydr. Chem. Biochem. 50: 211-276.

24. Raetz CR, Reynolds CM, Trent MS, Bishop RE. (2007) Lipid A Modification Systems in Gram-Negative Bacteria. Annu. Rev. Biochem. 76: 295-329.

25. Kucerka N, Papp-Szabo E, Nieh MP, Harroun TA, Schooling SR, Pencer J, Nicholson EA, Beveridge TJ, Katsaras J. (2008) Effect of Cations on the Structure of Bilayers Formed by Lipopolysaccharides Isolated From Pseudomonas Aeruginosa PAO1. J. Phys. Chem. B 112(27): 8057-8562.

26. Gronow S, Brade H. (2001) Invited Review: Lipopolysaccharide Biosynthesis: Which Steps Do Bacteria Need to Survive? J. Endotoxin Res. 7(1): 3-23.

27. Ediger MD. (2000) Spatially Heterogeneous Dynamics in Supercooled Liquids. Annu. Rev. Phys. Chem. 51(1): 99-128.

28. Chaudhuri P, Sastry S, Kob W. (2008) Tracking Heterogeneous Dynamics During the $\alpha$ Relaxation of a Simple Glass Former. Phys. Rev. Lett. 101(19): 190601.

29. Pink DA, Truelstrup Hansen L, Gill TA, Quinn BE, Jericho MH, Beveridge TJ. (2003) Divalent Calcium Ions Inhibit the Penetration of Protamine Through the Polysaccharide Brush of the Outer Membrane of Gram-Negative Bacteria. Langmuir 19(21): 88528858.

30. Clifton LA, Ciesielski F, Skoda MW, Paracini N, Holt SA, Lakey JH. (2016) The Effect of Lipopolysaccharide Core Oligosaccharide Size on the Electrostatic Binding of Antimicrobial Proteins to Models of the Gram-Negative Bacterial Outer Membrane. Langmuir 32(14): 3485-3494.

31. Hsu PC, Jefferies D, Khalid S. (2016) Molecular Dynamics Simulations Predict the Pathways via Which Pristine Fullerenes Penetrate Bacterial Membranes. J. Phys. Chem. B 120(43): 1117011179. 
32. Qiao R, Roberts AP, Mount AS, Klaine SJ, Ke PC. (2007)

Translocation of C60 and its Derivatives Across a Lipid Bilayer. Nano Lett. 7(3): 614-619.

33. D'Rozario RS, Wee CL, Wallace EJ, Sansom MS. (2009) The Interaction of C60 and its Derivatives with a Lipid Bilayer via Molecular Dynamics Simulations. Nanotechnology 20(11): 115102.

34. Wong-Ekkabut J, Baoukina S, Triampo W, Tang IM, Tieleman DP, Monticelli L. (2008) Computer Simulation Study of Fullerene Translocation through lipid membranes. Nat. Nanotechnol. 3(6): 363-368.

35. Kučerka N, Liu Y, Chu N, Petrache HI, Tristram-Nagle S, Nagle JF. (2005) Structure of Fully Hydrated Fluid Phase DMPC and DLPC Lipid Bilayers Using X-ray Scattering from Oriented Multilamellar Arrays and from Unilamellar Vesicles. Biophys. J. 88(4): 2626-2637.

36. Guler D, Ghosh DD, Pan J, Mathai JC, Zeidel ML, Nagle JF, Tristram-Nagle S. (2009) Effects of Ether vs. Ester Linkage on Lipid Bilayer Structure and Water Permeability. Chem. Phys. Lipids 160(1): 33-44.

37. Hub JS, De Groot BL, Van Der Spoel D. (2010) g_wham-a Free Weighted Histogram Analysis Implementation Including Robust Error and Autocorrelation Estimates. J. Chem. Theory Comput. 6(12): 3713-3720.

38. Carpenter TS, Parkin J, Khalid S. (2016) The Free Energy of Small Solute Permeation Through the E. coli Outer Membrane has a Distinctly Asymmetric Profile. J. Phys. Chem. Lett. 7(17): 34463451.

39. MacCallum JL, Tieleman DP. (2006) Computer Simulation of the Distribution of Hexane in a Lipid Bilayer: Spatially Resolved Free Energy, Entropy, and Enthalpy Profiles. J. Am. Chem Soc. 128(1): 125-130.

40. Schlitter J. (1993) Estimation of Absolute and Relative Entropies of Macromolecules Using the Covariance Matrix. Chem. Phys. Lett. 215(6): 617-621. 
41. Karplus M, Kushick JN. (1981) Method for Estimating the

Configurational Entropy of Macromolecules. Macromolecules 4(2): 325-332.

42. Oh Y, Kim J, Yethiraj A, Sung BJ. (2016) Swing Motion as a Diffusion Mechanism of Lipid Bilayers in a Gel Phase. Phys. Rev. E 93(1): 012409.

43. Klenk M, Lai W. (2015) Local Structure and Dynamics of Lithium Garnet Ionic Conductors: Tetragonal and Cubic Li7La3Zr2O7. Phys. Chem. Chem. Phys. 17(14): 8758-8768.

44. Lindhard J. (1965) Influence of Crystal Lattice on Motion of Energetic Charged Particles. Munksgaard;

45. Le Brun AP, Clifton LA, Halbert CE, Lin B, Meron M, Holden PJ, Lakey JH, Holt SA. (2013) Structural Characterization of a Model Gram-Negative Bacterial Surface Using Lipopolysaccharides from Rough Strains of Escherichia coli. Biomacromolecules 14(6): 20142022.

46. Jeworrek C, Evers F, Howe J, Brandenburg K, Tolan M, Winter R. (2011) Effects of Specific Versus Nonspecific Ionic Interactions on the Structure and Lateral Organization of Lipopolysaccharides.

Biophys. J. 100(9): 2169-2177.

47. Wei S, Gallino I, Busch R, Angell CA. (2011) Glass Transition with Decreasing Correlation Length During Cooling of Fe50Co50 Superlattice and Strong Liquids. Nat. Phys. 7(2): 178-182.

48. Angeli F, Villain O, Schuller S, Charpentier T, de Ligny D, Bressel L, Wondraczek L. (2012) Effect of Temperature and Thermal History on Borosilicate Glass Structure. Phys. Rev. B 85(5): 054110.

49. Errandonea D. (2009) Transition metals: Can Metals be a Liquid Glass? Nat. Mat. 8(3): 170-171.

50. Jurlewicz A, Meerschaert MM, Scheffler HP. (2011) Cluster Continuous Time Random Walks. Stud. Math. 205: 13-30.

51. Aichele M, Baschnagel J. (2001) Glassy Dynamics of Simulated Polymer Melts: Coherent Scattering and Van Hove Correlation Functions. Eur. Phys. J. E 5(2): 229-243. 
52. Kob W, Andersen HC. (1995) Testing Mode-Coupling Theory for a Supercooled Binary Lennard-Jones Mixture I: The Van Hove Correlation Function. Phys. Rev. E 51(5): 4626.

53. Risselada HJ, Marrink SJ, Müller M. (2011) Curvature-Dependent Elastic Properties of Liquid-Ordered Domains Result in Inverted Domain Sorting on Uniaxially Compressed Vesicles. Phys. Rev. Lett. 106(14): 148102.

54. Tristram-Nagle S, Nagle JF. (2004) Lipid Bilayers:

Thermodynamics, Structure, Fluctuations, and Interactions. Chem. Phys. Lipids 127(1): 3-14.

55. Ipsen JH, Mouritsen OG, Bloom M. (1990) Relationships Between Lipid Membrane Area, Hydrophobic Thickness, and Acyl-Chain Orientational Order. The Effects of Cholesterol. Biophys. J. 57(3): 405.

56. Lewis BA, Engelman DM. (1983) Lipid Bilayer Thickness Varies Linearly with Acyl Chain Length in Fluid Phosphatidylcholine Vesicles. J. Mol. Biol. 166(2): 211-217.

57. de Jong DH, Lopez CA, Marrink SJ. (2013) Molecular View on Protein Sorting Into Liquid-Ordered Membrane Domains Mediated by Gangliosides and Lipid Anchors. Farad. Discuss. 161: 347-363.

58. Lingwood D, Simons K. (2010) Lipid Rafts as a MembraneOrganizing Principle. Science 327(5961): 46-50.

59. Dykstra M, Cherukuri A, Sohn HW, Tzeng SJ, Pierce SK. (2003) Location is Everything: Lipid Rafts and Immune Cell Signaling*. Annu. Rev. Immunol. 21(1): 457-481.

60. Carruthers A, Melchior DL. (1988) Effects of Lipid Environment on Membrane Transport: the Human Erythrocyte Sugar Transport Protein/Lipid Bilayer System. Annu. Rev. Physiol. 50(1): 257-271.

61. Slater SJ, Kelly MB, Taddeo FJ, Ho C, Rubin E, Stubbs CD. (1994) The Modulation of Protein Kinase C Activity by Membrane Lipid Bilayer Structure. J. Biol. Chem. 269(7): 4866-4871.

62. Friedrich CL, Moyles D, Beveridge TJ, Hancock RE. (2000) Antibacterial Action of Structurally Diverse Cationic Peptides on 
Gram-Positive Bacteria. Antimicrob. Agents Chemother. 44(8): 2086-2092.

63. Straus SK, Hancock RE. (2006) Mode of Action of the New Antibiotic for Gram-Positive Pathogens Daptomycin: Comparison with Cationic Antimicrobial Peptides and Lipopeptides. Biochim. Biophys. Acta 1758(9): 1215-1223.

64. Hancock RE. (1997) Peptide Antibiotics. Lancet 349(9049): 418422.

65. Pronk S, Páll S, Schulz R, Larsson P, Bjelkmar P, Apostolov R, Shirts MR, Smith JC, Kasson PM, van der Spoel D, Hess B. (2013) GROMACS 4.5: a High-Throughput and Highly Parallel Open Source Molecular Simulation Toolkit. Bioinformatics btt055.

66. Marrink SJ, Risselada HJ, Yefimov S, Tieleman DP, De Vries AH. (2007) The MARTINI Force Field: Coarse Grained Model for Biomolecular Simulations. J. Phys. Chem. B 111(27): 7812-7824.

67. Parrinello M, Rahman A. (1981) Polymorphic Transitions in Single Crystals: A New Molecular Dynamics Method. J. Appl. Phys. 52(12): 7182-7190.

68. Parrinello M, Rahman A. (1980) Crystal Structure and Pair Potentials: A Molecular-Dynamics Study. Phys. Rev. Lett. 45(14): 1196.

69. Evans DJ, Holian BL. (1985) The Nose-Hoover Thermostat. J. Chem. Phys. 83(8): 4069-4074.

70. Chavent M, Reddy T, Goose J, Dahl AC, Stone JE, Jobard B, Sansom MS. (2014) Methodologies for the Analysis of Instantaneous Lipid Diffusion in MD Simulations of Large Membrane Systems. Farad. Discuss. 169: 455-475. 


\section{Biochemis $y \quad$ Page 24 of 24}

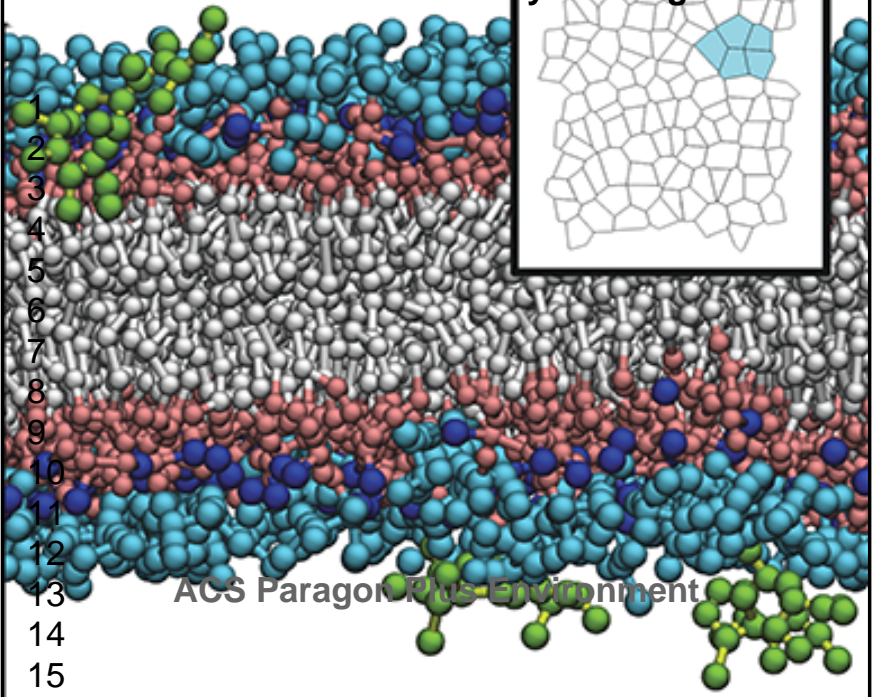

